\title{
VITAL-SIGN DATA FUSION MODELS FOR POST-OPERATIVE PATIENTS
}

\author{
Marco A. F. Pimentel ${ }^{1}$, David A. Clifton ${ }^{1}$, Lei Clifton ${ }^{1}$, Peter J. Watkinson ${ }^{2}$ and Lionel Tarassenko ${ }^{1}$ \\ ${ }^{1}$ Institute of Biomedical Engineering, Department of Engineering Science, University of Oxford, Oxford, U.K. \\ ${ }^{2}$ Nuffield Department of Anaesthetics, University of Oxford, Oxford, U.K.
}

Keywords: Patient Monitoring, Early Warning Scores, Novelty Detection.

\begin{abstract}
Deterioration in Patients who undergo upper-gastrointestinal surgery may be evident in the vital signs prior to adverse events. A dataset comprising observational vital-sign data from 128 post-operative patients was used to explore the trajectory of patients vital-sign changes during their stay in the post-operative ward. A model of normality based on pre-discharge data from patients who had a "normal" recovery was constructed using kernel density estimates, and tested with "abnormal" data from patients who deteriorate sufficiently to be re-admitted to the Intensive Care Unit. The results suggest that the criticality of post-operative patients can be evaluated by assessment of the distributions of their vital signs after their admission to the post-operative ward.
\end{abstract}

\section{INTRODUCTION}

A large number of preventable and avoidable deaths occur in hospitals each year in the UK, due to adverse events such as cardiac arrest and unplanned admission into the ICU from other hospital wards (Bardell et al., 2003; Kause et al., 2004). Patients who undergo surgery for the removal of upper- gastrointestinal (GI) cancer have a high incidence of post-operative complications, often resulting in readmission to the Intensive Care Unit (ICU) several days after surgery (Thompson et al., 2003; Veltkamp et al., 2002). Failure to identify such deteriorations in a timely manner has led to the design of a two-phase clinical trial at the Oxford Cancer Hospital, the "CALMS2" trial, in which ambulatory post-operative cancer patients are monitored using wearable sensors that are connected by the hospital wireless network to patient monitors and nursing display stations. Vital-sign measurements are also made periodically by the nurses on the ward as part of the usual post-operative care.

Our approach to monitoring in-hospital patients relies on constructing models of normality based on the vital signs acquired from a large population of acutely-ill patients (Tarassenko et al., 2006; Hann, 2008). We propose to improve upon existing models by investigating models tuned to specific patient populations. Post-operative cancer patients are recovering from surgery, so that they start in their most acute state and then gradually stabilise. In this work, we therefore adopt a novel approach in which the aim is to learn the vital-sign trajectories associated with "normal" recovery of these patients, allowing "abnormal" trajectories to be identified in subsequent patients.

We present preliminary results of the analysis of data acquired during Phase I of the CALMS2 clinical trial. A multivariate model of the distribution of vitalsign data from "normal" patients, which describe the normal physiological trajectory, is constructed using a non-parametric statistical approach, a kernel density estimate. This model is then tested on "abnormal" data from patients who deteriorate sufficiently after surgery to be re-admitted to the ICU.

\section{DATASET}

The dataset used for the work described by this paper comprises measurements of heart rate (HR), respiratory rate (RR), peripheral arterial oxygen saturation $\left(\mathrm{SpO}_{2}\right)$, systolic blood pressure (SBP) and temperature acquired by ward staff every hour or every two hours in the first days after patient admission (depending on the patient's condition), and every four hours in the last days of the patient's stay on the ward. A set of 15,029 observations $\mathbf{X} \in \mathbb{R}^{5}$ was obtained from 169 patients. These patients were admitted to 
the Upper GI ward and the median length of stay on the ward was 8 days (IQR 6-11). In order to select a subgroup of patients with "normal" recovery from surgery (class $C_{1}$ ), we chose those patients who stayed more than 4 days and less than 25 days on the ward, and who were not (re-)admitted to the ICU. This resulted in 128 patients being included in class $C_{1}$.

It is assumed, a priori, that each of the $D=5$ parameters has equal importance in the patient model. Each parameter was therefore scaled to have the same dynamic range using a zero-mean, unit-variance transformation.

The vital-sign trajectory throughout patient stays on the ward was evaluated by examining the 5 subgroups of data:

- $G_{1}$ : comprises the first clinical observation performed when a patient was admitted to the ward;

- $G_{2}$ : is the average of all observations performed on the first day of a patient's stay on the ward;

- $G_{3}$ : comprises the average of all observations performed on the day that corresponds to half of the length of the patient's stay on the ward (if the patient stayed 5 days on the ward prior to discharge, this day would correspond to day three);

- $G_{4}$ : contains the average of all observations performed on the day that corresponds to $75 \%$ of the length of the patient's stay on the ward;

- $G_{5}$ : contains the average of all observations performed on the last day of the patient's stay on the ward.
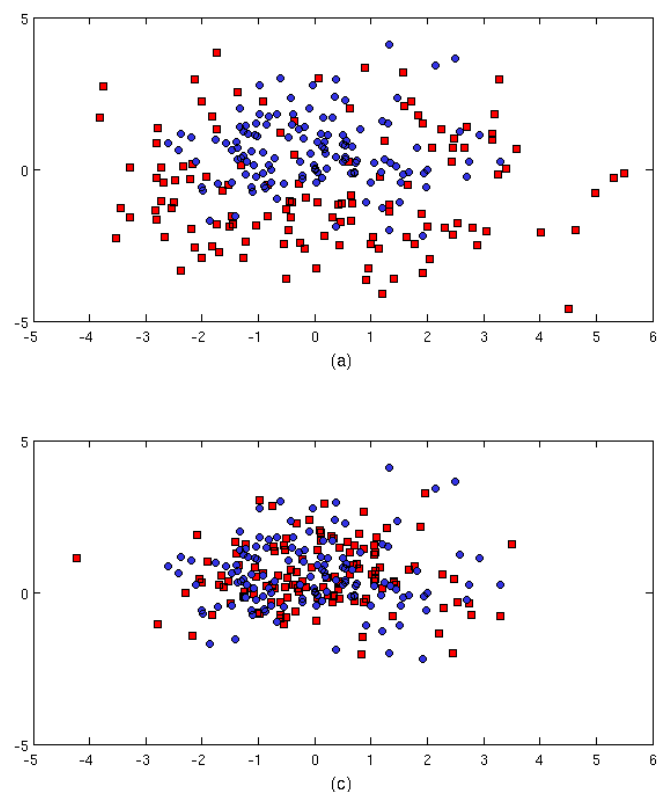

These five subgroups were defined in this way because of the different lengths of patient stay on the ward (from 4 to 25 days).

\section{DATA VISUALISATION}

\subsection{Methodology}

The first stage in constructing a model of normality for novelty detection usually consists in obtaining more insight into the structure of the data. Procedures for visualisation of the data in their original high-dimensional space are required. The relations of proximity of the vital-sign data in their original space, with $D>3$, can be visualised through a non-linear projection from $\mathbb{R}^{D}$ to $\mathbb{R}^{3}$ or $\mathbb{R}^{2}$.

A Sammon-mapping algorithm (Sammon, 1969) was implemented and applied in the 640 mean vectors contained in the 5 subgroups $\left(G_{1}, G_{2}, G_{3}, G_{4}\right.$, $G_{5}$, with 128 data in each) from patients belonging to class $C_{1}$.

\subsection{Results}

Represented in each map of Figure 1 are the projected data from $G_{1}, G_{2}, G_{3}, G_{4}$ superimposed on the projected data points from $G_{5}$ (data from the last day on the ward for each patient).

It may be seen from the plots that the groups form
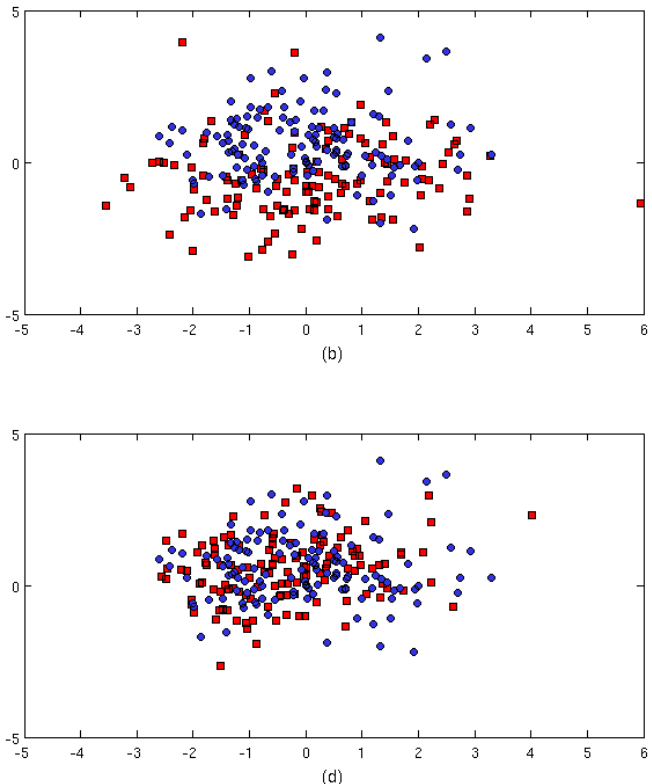

Figure 1: Sammon maps obtained for the group dataset: (a) $G_{1}$ and $G_{5}$, (b) $G_{2}$ and $G_{5}$, (c) $G_{3}$ and $G_{5}$, and (d) $G_{4}$ and $G_{5}$; in (a)-(d) $G_{1}, G_{2}, G_{3}$ and $G_{4}$ are represented by red $\{\square\}$ and $G_{5}$ by blue $\{0\}$. 
clusters with some overlap between them. If we consider projected data from groups $G_{1}$ and $G_{5}$ (shown in red and blue, respectively in the top-left plot), it may be seen that the former are dispersed, while the latter are more concentrated. It may also be seen that the distributions of the data for $G_{3}$ (data that correspond to the midpoint of patient's stay) and $G_{5}$ groups are very similar and evenly concentrated. This suggests that for the 128 patients with a normal recovery process from surgery, there are no large changes in the vital-sign distributions after the midway point during their stay on the ward. These results suggest the possibility that patients belonging to class $C_{1}$ could have been discharged early, or provided with a lower level of care from the halfway point of their stay, as they had already been stabilised and no significant changes in their vital-sign distributions subsequently occured.

\section{MODEL OF NORMALITY}

\subsection{Methodology}

For the construction of the model of normality, we considered the $G_{5}$ subgroup of measurements as the "normal" dataset. This contains the most stable physiological variables (because these data are acquired just before discharge, when the patient is most "normal" ). At discharge the patient is stable and deemed well enough to go home. Therefore, the $G_{5}$ dataset of $N=128$ prototype vectors, $\mathbf{X} \in \mathbb{R}^{5}$, was used for construction of the model of normality.

A kernel density estimate (Bishop, 2007) is a technique that allows the underlying 5-D vital-sign probabilty density function (pdf) to be estimated from training data. While other methods, such as Gaussian mixture models, were considered, a kernel density estimate was chosen as it has the advantage of being a fully non-parametric method, so no a priori assumptions are made about the form of the probability distribution.

This method was initially used to estimate the pdf of the 128 prototype vectors, $\mathbf{x}_{1}, \ldots, \mathbf{x}_{\mathbf{N}}$,

$$
p(\mathbf{x})=\frac{1}{\mathbf{N}(\mathbf{2} \pi)^{\mathbf{D} / 2} \sigma^{\mathbf{D}}} \sum_{i=1}^{\mathbf{N}} \mathbf{e}^{-\frac{\left|\mathbf{x}-\mathbf{x}_{i}\right|^{2}}{2 \sigma^{2}}}
$$

which is a weighted sum of Gaussian kernels centred on the 128 prototype vectors, $\mathbf{x}_{i}$, and where each kernel is isotropic with variance $\sigma^{2}$. The variance was determined using the nearest-neighbour method proposed by Bishop (Bishop, 2007).

In the final model, only those patients who were discharged within 5 to 9 days were considered for building the estimate of the pdf $(N=78)$. The rationale for this decision was to focus on the most "normal" patients from class $C_{1}$ (i.e. the patients who recover entirely as expected after surgery). To identify those patients, the mode of the lengths of stay on the ward was calculated, and only the patients whose stay on the ward was within the mode \pm 2 days were taken into account. This corresponds to the range [5 9] days. The averages of the observations from each day for each of these patients were calculated and the pdfs $p(\mathbf{x} \mid \theta)$, where $\theta$ corresponds to the model parameters, were then determined from Equation (1) with $N=78$.

In order to estimate the abnormality of a previously-unseen test pattern, the departure from normality is usually quantified using novelty defined as $z(\mathbf{x})=-\log p(\mathbf{x} \mid \theta)$, where $z(\mathbf{x})$ is the novelty score. This process leads to "normal" data, which have higher likelihoods $p(\mathbf{x} \mid \theta)$, generating low novelty scores, and "abnormal" data, which have lower likelihoods, generating high novelty scores.

\subsection{Results}

The novelty scores $z(\mathbf{x})$ from the most "normal" 78 patients using the 5-D pre-discharge data $\left(G_{5}\right)$ to construct the model of normality are shown in Figure 2, averaged for each patient for each day. These scores confirm the results given by the Sammon maps. From the trajectory of the novelty $z(\mathbf{x})$ shown in Figure 2 we can conclude that there is a significant decrease of the value of $z(\mathbf{x})$ during the first 3 days. From day 4 onwards, $z(\mathbf{x})$ remains approximately constant. The first 3 days could be associated with the patient's recovery in the first post-operative days (Thompson et al., 2003), whereas after day 4, the majority of patients

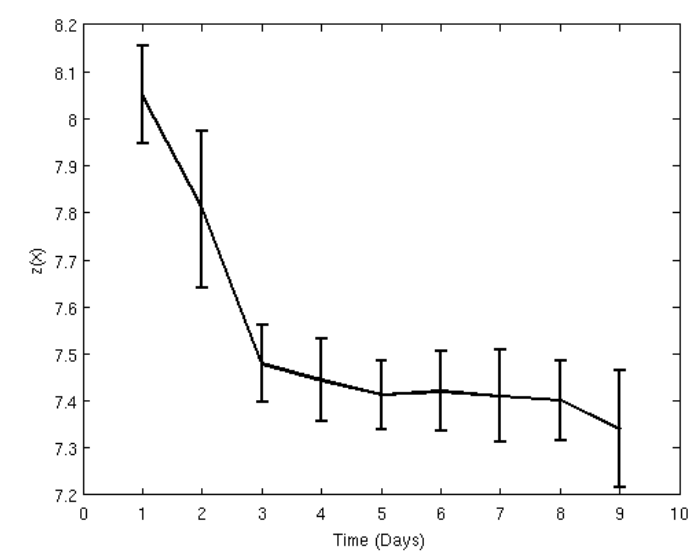

Figure 2: Representation of average of the novelty scores $z(\mathbf{x})=-\log p(\mathbf{x} \mid \theta)$ with time for "normal" patients, where the data probability density was constructed using predischarge data from the same group of patients. Error bars denoted one standard error of the group mean. 
included in this study appear to have fully recovered from surgery and to be stable. They are well enough to either be discharged or be provided with a lower level of care for their remaining time on the ward.

The multivariate model of normality was also tested on "abnormal" vital-sign data from two patients who deteriorated sufficiently after surgery to be readmitted to the ICU. The model was able to identify patient deterioration up to 12 hours before the patient's readmission to the ICU. These results are omitted for brevity.

\section{ACKNOWLEDGEMENTS}

The work described in this paper was funded by the NIHR Biomedical Research Centre Programme, Oxford. MAFP was supported by the RCUK Digital Economy Programme grant number EP/G036861/1 (Oxford Centre for Doctoral Training in Healthcare Innovation), and Dr David Clifton was supported by the Wellcome Trust and the EPSRC under grant number WT $088877 / \mathrm{Z} / 09 / \mathrm{Z}$. The authors also wish to thank the support of all clinical staff involved in the collection of the data used in this investigation.

\section{CONCLUSIONS AND FUTURE WORK}

We have presented the preliminary analysis of data acquired from patients who were admitted to the post-operative Upper GI ward after cancer surgery. We studied the vital-sign distributions at the time of the patient's admission to the ward, halfway through their stay, and near the time of discharge. Although changes in vital-sign distributions from "normal" patients between admission to the ward and subsequent discharge were found, no significant changes in these distributions were observed from halfway through their stay to the time of discharge, which suggests that these patients could have been discharged earlier or provided with a lower level of care from the halfway point of their stay on the ward.

A multivariate model of the distribution of vitalsign data from "normal" patients, which describe the normal trajectories, was constructed using a kernel density estimate. The model confirmed the results obtained with the Sammon maps.

Future work will concentrate on the refinement of existing techniques for the target population group, and on the improvement of model construction using more complex dynamical modelling methods. However, there are a number of limitations that must be overcome in future analysis. The dataset used in the analysis described by this paper consisted of measurements of vital signs acquired periodically (every 2 or 4 hours) by ward staff during Phase I of the clinical trial. These infrequent patient observations combined with lower nurse:patient ratios (typically, 1:4 to 1:10 in step-down wards), can lead to unnoticed clinical deterioration and, consequently, to increased numbers of adverse events. We are developing a continuous vital-sign monitoring system to provide early warning of patient deterioration in a robust manner with low numbers of false alarms.

\section{REFERENCES}

Bardell, T., Legare, J. F., Buth, K. J., Hirsch, G. M., and Ali, I. S. (2003). ICU readmission after cardiac surgery. European Journal of Cardio-Thoracic Surgery, 23(3):354-359.

Bishop, C. M. (2007). Pattern Recognition and Machine Learning. Springer.

Hann, A. (2008). Multi-parameter monitoring for early warning of patient deterioration. Ph.D. thesis, University of Oxford.

Kause, J., Smith, G., Prytherch, D., Parr, M., Flabouris, A., and Hillman, K. (2004). A comparison of antecedents to cardiac arrests, deaths and EMergency intensive care admissions in australia and new zealand, and the united kingdom-the ACADEMIA study. Resuscitation, 62(3):275-282.

Sammon, J. W. (1969). A nonlinear mapping for data structure analysis. IEEE Transactions on Computers, C18(5):401- 409.

Tarassenko, L., Hann, A., and Young, D. (2006). Integrated monitoring and analysis for early warning of patient deterioration. British Journal of Anaesthesia, 97(1):64 -68 .

Thompson, J. S., Baxter, B. T., Allison, J. G., Johnson, F. E., Lee, K. K., and Park, W. Y. (2003). Temporal patterns of postoperative complications. Arch Surg, 138(6):596-603.

Veltkamp, S. C., Kemmeren, J. M., van der Graaf, Y., Edlinger, M., and van der Werken, C. (2002). Prediction of serious complications in patients admitted to a surgical ward. The British Journal of Surgery, 89(1):94-102. 